\title{
A Secure Routing Protocol for Cluster-based Wireless Sensor Networks Using ID-based Digital Signature
}

\author{
Huang Lu, Jie Li, and Hisao Kameda \\ Dept. of Computer Science, Univ. of Tsukuba, Tsukuba Science City, 305-8573, Japan \\ Email: luhuang@osdp.cs.tsukuba.ac.jp, lijie@cs.tsukuba.ac.jp, kameda@cs.tsukuba.ac.jp
}

\begin{abstract}
In this paper, we study the secure routing for clusterbased sensor networks where clusters are formed dynamically and periodically. We point out the deficiency in the secure routing protocols with symmetric key pairing. Along with the investigation of ID-based cryptography for security in WSNs, we propose a new secure routing protocol with ID-based signature scheme for cluster-based WSNs, in which the security relies on the hardness of the Diffie-Hellman problem in the random oracle model. Because of the communication overhead for security, we provide analysis and simulation results in details to illustrate how various parameters act between security and energy efficiency.

Index Terms-wireless sensor networks, cluster-based, IDbased digital signature, secure routing protocol.
\end{abstract}

\section{INTRODUCTION}

A wireless sensor network (WSN) is a network system comprising spatially distributed devices using wireless sensor nodes to monitor physical or environmental conditions, such as sound, temperature, and motion. In a WSN, the individual nodes are capable of sensing their environments, processing the information data locally, and sending data to one or more collection points through a wireless link [1]. Sensor nodes are severely constrained by the energy from batteries, which limits the lifetime and quality of the network. Therefore, it is crucial for WSNs to maximize nodes' lifetimes, reduce bandwidth consumption by using local collaboration among sensor nodes |2|. Many sensor systems are deployed in harsh, and often adversarial physical environments, such as battle fields and military domains with trustless surroundings [3]. Therefore, security is vastly requested in many practical WSNs.

Grouping sensor nodes into clusters has been widely investigated by researchers in order to achieve the network system's scalability and management objectives. Every cluster would have a leader sensor node, often referred to as the cluster-head $(\mathrm{CH})$, which can be fixed or variable. $\mathrm{CHs}$ aggregate the data collected by the sensor nodes in its cluster, thus, clustering decreases the number of relayed packets. As the benefits of clustering, it conserves communication bandwidth and avoids redundant exchange of messages among sensor nodes, because of limiting the inter-cluster interactions. Therefore, clustering prolongs the lifetime of WSNs |2||4|.

Digital signature is one of the most critical security services offered by cryptography. In traditional key management systems, the binding between the public key and the identification of the signer is obtained via a digital certificate $[5]$. As noticed by Shamir [6], it would be more efficient if there is no need for this binding, in which the users identity would be their public key. Moreover, given the user's identity, the public key could be easily derived using some public specified algorithms. An identity based (ID-based) signature scheme, whose security is based on the difficulty of factoring integers, is given by Hess [7], and it remained an open issue to develop an efficient IDbased encryption scheme.

In this paper, we focus on efficient secure communications, and propose a secure routing protocol using ID-based digital signature for cluster-based WSNs. In the proposed protocol, $\mathrm{CHs}$ are selected autonomously and communicate with the base station (BS) directly, whereas leaf sensor nodes join a cluster depending on the strength of transmission signal. The secure routing protocol is based on the ID-based cryptography, in which users' public keys are their ID information, and users can obtain the corresponding private keys without auxiliary data transmission. Therefore, the secure protocol is efficient in communication. However, it suffers from high-computation cost for pairing. We analyze the positive and negative aspects of the proposed routing protocol quantitatively in calculations and simulations, with respect to both computational and communication cost.

In section II and III, we provide an overview of related work and preliminary knowledge related to this work. In section IV, we describe the features and the operation of the proposed protocol, whereas security vulnerabilities and objectives. In section $\mathrm{V}$, we propose an ID-based secure routing protocol for cluster-based WSNs. In section VI, we analyze the proposed secure routing protocol with calculations and simulations. Section VII offers conclusion and points of future work.

\section{RELATED WORK}

In this section, we present the related work including the cluster-based routing protocols, security issues, and ID-based cryptography.

The LEACH (Low-Energy Adaptive Clustering Hierarchy) protocol presented by Heinzelman et al. $|8|$ is a widely known and effective one for WSNs of cluster-based architecture. It assumes that every node can directly reach the BS by transmitting with high enough energy. However, leaf sensor nodes (non- $\mathrm{CH}$ sensor nodes) send the sensed data to their $\mathrm{CHs}$ in order to save energy, which then aggregate the data, and send the aggregation to the BS. To prevent quick energy consuming of the set of $\mathrm{CHs}$, LEACH randomly rotates $\mathrm{CHs}$ among all sensor nodes in the network, from time to time in rounds. LEACH achieved improvements in terms of nodes' lifetime. 
Following the idea of LEACH, a number of protocols have been presented such as PEGASIS (Power-Efficient Gathering in Sensor Information Systems) [9], and PEACH (Powerefficient and adaptive clustering hierarchy protocol for wireless sensor networks) $[10 \mid$, which use the similar concept with LEACH. In this paper, for convenience, we call this sort of cluster-based routing protocols as LEACH-like protocols.

Because traditional privacy protection cannot solve security vulnerability in WSNs, some security protocols have been proposed towards WSNs, e.g., SPINS (Security Protocols for Sensor Network) [11]. Adding security to LEACH-like protocols is challenging, because its dynamic clustering makes key distribution solutions for node-to-node trust relationships messy and inadequate. There are some secure routing protocols based on LEACH-like protocols, such as SecLECH [12] and GS-LEACH |13|. However, most of them are applying symmetric key management scheme, which increases the overhead of transmission by establishing pairwise keys. For example, SecLEACH has such a problem that if a node does not share a pairwise key with others in its preloaded key ring, then it cannot participate in any cluster and has to elect itself as a $\mathrm{CH}$. We call it the orphan node problem and this problem increases the overhead of transmission.

As the evolution of the traditional certificate-based cryptography, ID-based cryptography (IBC) has been receiving high regard since the idea of IBC was proposed in 1984 [6]. The main idea is to derive an entity's public key from its identity information, e.g., from its name or the ID number. However, the fully-functional ID-based encryption scheme had not been found until Boneh and Franklin [5] found it in 2001. The scheme given by them becomes popular, which is based on the bilinear Diffie-Hellman problem being related to a pairing algorithm |14|. Furthermore, the authentication scheme using digital signature, which applies IBC, has been proposed in |7|. Recently, the concept of IBC has been rapidly developed in WSN systems. Carman in 2005 first combined the benefits of IBC and key pre-distribution and sets into WSNs [15].

\section{Preliminaries}

The preliminary knowledge of pairing technique and parameters used in IBC are addressed below.

In the proposed secure routing protocol, the security is based on the Bilinear Diffie-Hellman Problem (BDHP) in the pairing domain. Therefore, the Discrete Logarithm Problem (DLP) [16] for pairing in groups is required to be hard. Randomly select two large primes $p$ and $q$, and let $\mathbb{E} / \mathbb{F}_{p}$ indicate an elliptic curve $y^{2}=x^{3}+a x+b\left(4 a^{3}+27 b^{2} \neq 0\right)$ over the finite field $\mathbb{F}_{p}$. We denote by $\mathbb{G}_{1}$ a $q$-order subgroup of the additive group of points in $\mathbb{E} / \mathbb{F}_{p}$, and $\mathbb{G}_{2}$ a $q$-order subgroup of the multiplicative group in the finite field $\mathbb{F}_{p}^{*}$. The pairing is a mapping $e: \mathbb{G}_{1} \times \mathbb{G}_{1} \rightarrow \mathbb{G}_{2}$, which is a bilinear map if it satisfies the following properties:

1) Bilinear: For $\forall P, Q, R, S \in \mathbb{G}_{1}, e(P+Q, R+S)=$ $e(P, R) e(P, S) e(Q, R) e(Q, S)$. In the same way, for $\forall c, d \in \mathbb{Z}_{q}^{*}$, we have $e(c P, d Q)=e(P, d Q)^{c}=$ $e(c P, Q)^{d}=e(P, Q)^{c d}$, etc.
2) Non-degeneracy: If $P$ is a generator of $\mathbb{G}_{1}$, then $e(P, P)$ is a generator of $\mathbb{G}_{2}$.

3) Computability: There is an efficient algorithm to compute $e(P, Q)$ in $\mathbb{G}_{2}$, for $\forall P, Q \in \mathbb{G}_{1}$.

Weil pairing and Tate pairing are the examples of such bilinear mapping, the comprehensive description of how pairing parameters be selected for security, refers to $|5|$ and $|14|$.

\section{System Description and Security Objectives}

This section introduces the network structure and the operation mechanism. After that, security objectives are presented.

\section{A. Network Architecture and Operation Mechanism}

In this paper, we consider a WSN system that consists of a fixed base station and a large number of sensor nodes, which are homogeneous in functionalities and capabilities. We suppose that, the sink is always reliable, but sensor nodes are subject to be compromised by the attackers. In this WSN system, data are sensed by sensor nodes and transmitted to a base station via $\mathrm{CHs}$, which perform data fusion. We assume that all sensor nodes and the BS use symmetric radio channel, that nodes are distributed randomly, and that their energy is constrained.

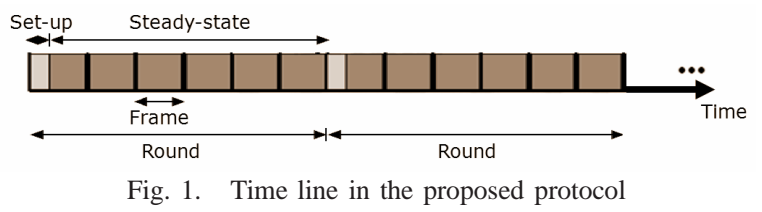

The operation of the protocol is divided by rounds as shown in Figure 1. Each round includes a set-up phase for constructing clusters and a steady-state phase for transmitting data from sensor nodes to the BS via CHs. In each round, the algorithm elects CHs in the set-up part, and in each frame, a sensor node transmits the data to its $\mathrm{CH}$.

We use the dynamic clustering method from LEACH [8] to all sensor nodes to increase energy efficiency. For fair energy consumption, nodes are randomly elected as $\mathrm{CHs}$ in rounds. With $\mathrm{CHs}$, other non-CH sensor nodes join clusters using onehop transmission. In order to elect CHs in a round, each sensor node determines a random number and compare it with the threshold in the current round.

\section{B. Security Vulnerabilities and Objectives}

Like other type routing protocols in WSNs, cluster-based routing protocols (LEACH-like protocols) are vulnerable to a number of security attacks [17], e.g., jamming, spoofing, replay, etc. Because data aggregation and routing depend on the $\mathrm{CHs}$ fundamentally, attacks involving $\mathrm{CHs}$ could be the serious damaging to the network system. If an attacker manages to turn into a $\mathrm{CH}$, it can provoke attacks such as sinkhole and selective forwarding attacks, thus disrupting the network. On the other hand, the attacker may intend to inject bogus sensing data into the network, especially to pretend as leaf nodes sending bogus information towards the $\mathrm{CHs}$ [12]. Another type of attack is network eavesdropping. Nevertheless, 
LEACH-like protocols are more robust against insider attacks than most other types of routing protocols [17]. Because $\mathrm{CHs}$ in LEACH are the intermediary nodes to the BS, which change from time to time in rounds. The characteristics in LEACH-like protocols reduce the risks of being attacked on intermediary nodes, and make it harder for an adversary to identify and compromise important nodes.

\section{Proposed Secure Routing Protocol for Cluster-BAsed WSNS}

This section describes the operation details in the proposed routing protocol with the ID-based authentication.

\section{A. Protocol Initialization}

In the proposed ID-based secure routing protocol, time is divided into successive time intervals, which are denoted by time-stamps $T_{s}$ (for BS-to-node transmission) and $t_{i}$ (for leafto-CH transmission). We assume that the BS and sensor nodes are synchronized. We then adopt $I D \| t_{i}$ as user's public key for ID-based signature. In this way, before a sensor node wants to authenticate itself to another node, it has to obtain its private key first. Because each private key is valid only during the current time interval, sensor nodes have to obtain a denotation of the new time interval to renew the private key at the beginning of a new round. Upon node revocation, the BS needs to broadcast the compromised node IDs to the sensor nodes, each node stores the revoked IDs within the certain round.

Before the network deployment, the BS does the following operations of key predistribution in the network:

1) Generate the pairing parameters $\left(p, q, \mathbb{E} / \mathbb{F}_{p}, \mathbb{G}_{1}, \mathbb{G}_{2}, e\right)$, as described in Section III. Select a generator $P$ of $\mathbb{G}_{1}$ stochastically.

2) Choose two cryptographic hash functions: $H$, for point mapping hash function which maps strings to elements in $\mathbb{G}_{1}$, and $h$, which maps arbitrary inputs to fixed-length outputs, e.g., SHA-1 [18].

3) Pick a random integer $\tau \in \mathbb{Z}_{q}^{*}$, and set $P_{p u b}=\tau P$ as a network public key.

4) Preload each sensor node with the public system parameters $\left(p, q, \mathbb{E} / \mathbb{F}_{p}, \mathbb{G}_{1}, \mathbb{G}_{2}, e, H, h, P, \tau\right)$.

\section{B. Proposed Secure Routing}

The proposed secure routing protocol operates in rounds based on LEACH. Each round has a set-up phase and a steadystate phase. Because of the synchronization of time, sensor nodes know when each round starts and ends. We first address the key management of ID-based cryptography for secure routing, then introduce the operation of the routing protocol.

1) Key management for security:

Assume that user $j$ wants to broadcast a message $M$. It first obtains its private key as $S K_{j}=\tau H\left(I D \| t_{i}\right)$, where $I D_{j}$ is the ID of node $j$, and $t_{i}$ is the time-stamp of the time interval in the current round from TDMA (time division multiple access) control. The sensor then picks a random $\alpha \in \mathbb{Z}_{q}^{*}$ and computes $\theta=e(P, P)^{\alpha}$. The sensor node further computes

$$
c_{j}=h\left(M\left\|t_{i}\right\| \theta\right),
$$

and let

$$
\sigma_{j}=c_{j} S K_{j}+\alpha P .
$$

where $\left\langle\sigma_{j}, c_{j}\right\rangle$ is the digital signature on the message $M$. The broadcast message is now concatenated in the form of

$$
\left\langle I D_{j}, t_{i}, M, \sigma_{j}, c_{j}\right\rangle .
$$

Upon receiving the message in (3), each sensor node verifies the authenticity in the following way: It checks the time-stamp of current time interval $t_{i}$ and determines whether the received message is fresh. Then, if the time-stamp is correct, the sensor node further computes,

$$
\theta^{\prime}=e\left(\sigma_{j}, P\right) e\left(H\left(I D_{j} \| t_{i}\right),-P_{\text {pub }}\right)^{c_{j}} .
$$

Using the time-stamp of current time interval $t_{i}$ and a random $\alpha$ for deriving $\theta^{\prime}$, the sensor node does the bilinear transformation and mapping from $\theta^{\prime}$ to $\theta$. We should have the formula below if the received message is authentic:

$$
\begin{aligned}
\theta^{\prime} & =e\left(\sigma_{j}, P\right) e\left(H\left(I D_{j} \| t_{i}\right),-P_{p u b}\right)^{c_{j}} \\
& =e\left(\sigma_{j}, P\right) e\left(H\left(I D_{j} \| t_{i}\right),-\tau P\right)^{c_{j}} \\
& =e\left(c_{j} S K_{j}+\alpha P, P\right) e\left(H\left(I D_{j} \| t_{i}\right), \tau P\right)^{-c_{j}} \\
& =e\left(c_{j} S K_{j}+\alpha P, P\right) e\left(\tau H\left(I D_{j} \| t_{i}\right), P\right)^{-c_{j}} \\
& =\left(e\left(S K_{j}, P\right)^{c_{j}} e(P, P)^{\alpha}\right) e\left(\tau H\left(I D_{j} \| t_{i}\right), P\right)^{-c_{j}} \\
& =e\left(S K_{j}, P\right)^{c_{j}} e(P, P)^{\alpha} e\left(S K_{j}, P\right)^{-c_{j}} \\
& =e(P, P)^{\alpha} \\
& =\theta
\end{aligned}
$$

Then, if $h\left(M\left\|t_{i}\right\| \theta^{\prime}\right)=h\left(M\left\|t_{i}\right\| \theta\right)=c_{j}$, which is equal to that in the received message, the sensor node considers the received message authentic, and propagates the message to the next hop or user. If the verification above fails, the sensor node considers the message as a bogus or replaced one, even a mistaken one, and ignores it.

2) Operation of routing protocol:

Those who decide to become CHs broadcast an advertisement message $(a d v)$ to all sensor nodes in the network, which is concatenated with the digital signature. In step 3, the nodes that decide to be leaf nodes pick a $\mathrm{CH}$ to join in, which based on the largest received signal strength of $a d v$ messages, then, communicate with the $\mathrm{CH}$ by sending a join request (join) message. After the processes above, in step 4, the $\mathrm{CHs}$ broadcast an allocation message to their cluster members for communication during the steady-state phase, which includes a time slot schedule (sched) by TDMA control, yet to be concatenated with the digital signature.

Table I shows the whole operations in the proposed secure routing protocol. The set-up phase consists of four steps, from step 1 to 4 . During the set-up phase of a new round, the BS first broadcasts its ID and the denotation of the start time of the current round $T_{s}$, and a nonce (number used once) to all sensor nodes. Then the sensor nodes decide whether to become a $\mathrm{CH}$ for the current round, based on the threshold $T(n)$, and 
Table I. Operation of the protocol

\section{Set-up phase}

$$
\begin{aligned}
& \text { 1. } B S \Rightarrow G_{s} \quad:\left\langle I D_{B S}, T_{s}, \text { nonce }\right\rangle \\
& \text { 2. } C H_{i} \Rightarrow G_{s} \quad:\left\langle I D_{i}, T_{s}, a d v, \sigma_{i}, c_{i}\right\rangle \\
& \text { 3. } L_{j} \rightarrow C H_{i} \quad:\left\langle I D_{j}, T_{s}, I D_{j}, \text { join, } \sigma_{j}, c_{j}\right\rangle \\
& \text { 4. } \mathrm{CH}_{i} \Rightarrow G_{s} \quad:\left\langle I D_{i}, T_{s}, \operatorname{sched}\left(\ldots, I D_{j} / t_{i}, \ldots\right), \sigma_{i}, c_{i}\right\rangle \\
& \text { 5. } L_{j} \rightarrow \mathrm{CH}_{i} \quad:\left\langle I D_{j}, t_{i}, M, \sigma_{j}, c_{j}\right\rangle \\
& \text { 6. } C H_{i} \rightarrow B S \quad:\left\langle I D_{i}, T_{s}, F, \sigma_{i}, c_{i}\right\rangle \\
& \Rightarrow, \rightarrow \quad \text { : Broadcast and unicast transmission. } \\
& L_{j}, C H_{i}, G_{s} \quad \text { : A leaf node, a cluster head, and the set of sensor } \\
& T_{s}, t_{i} \text { : Time-stamps of the time slot for transmission. } \\
& \text { ID : The ID of a sensor node. } \\
& M, F \quad \text { : Sensed data and aggregated data. } \\
& \text { adv,join,sched : Message string types. } \\
& \langle\sigma, c\rangle \quad \text { : The digital signature concatenated with data. }
\end{aligned}
$$

compare the with the pre-determined random number from 0 to 1 , which is set as follows:

$$
\begin{aligned}
& T(n)=\frac{\rho}{1-\rho \times\left(r \bmod \left\lfloor\frac{1}{\rho}\right\rfloor\right)} \quad \forall n \in G_{n} \\
& T(n)=0 \quad \forall n \notin G_{n}
\end{aligned}
$$

$\rho$ is a priori determined value which represents the desired percentage of $\mathrm{CHs}$ during a round (e.g. $\rho=10 \%$ ), $r$ is the round number of the current round, and $G$ is the set of nodes that have not been $\mathrm{CH}$ nodes in the last $\lfloor 1 / \rho\rfloor$ rounds. If the value of determined number is less than the threshold, the sensor node elects itself as a $\mathrm{CH}$.

Once set-up phase is over, the network system turns into the steady-state phase, in which sensed data are transmitted from sensor nodes to the BS. First, according to the time slot schedule in step 4, each leaf sensor node $j$ transmits the data $M$ in a packet $\left\langle I D_{j}, t_{i}, M, \sigma_{j}, c_{j}\right\rangle$ to its $\mathrm{CH}$, which is concatenated with a digital signature in a time slot. Then, each $\mathrm{CH}$ sensor node collects messages from all members in its cluster, aggregates and fuses these data. Finally, in step 6, CHs send the aggregated data $F$ to the BS. The steady-state phase consists of multiple reporting cycles of data transmissions from leaf nodes to the $\mathrm{CHs}$, and is exceedingly long compared to the set-up phase.

\section{Security Analysis}

The proposed secure routing protocol provides different types of security services to the communications, in both set-up phase and steady-state phase. The encryption of the message provides confidentiality, the hash function provides integrity, using time interval time-stamps and the nonce provides freshness. Thus on the whole, ID-based digital signature provides confidentiality, authenticity, non-repudiation, integrity and freshness. Using the ID-based digital signature authentication has at least two advantages: First, it eliminates the utilizing of certificate and auxiliary authentication information. Also, because only the compromised node IDs have to be stored, it requires very small storage space for the nodes' revocation, since the length of a user ID is only 2 bytes. However, the disadvantages of this ID-based digital signature are also obvious because of the high-computation cost for bilinear transformation in pairing.

Focusing on the resilience to the certain attacks in clusterbased WSNs, such as sinkhole attacks, hello flood attacks and selective forwarding attacks, the routing protocol works well. All the three kinds of attacks are pointed to $\mathrm{CHs}$ of acting as intermediary nodes, because the leaf nodes make no sense of being attacked in cluster-based structure. Since attackers do not have valid digital signature to concatenate with broadcast messages for authentication, attackers cannot pretend as the $\mathrm{BS}$ or $\mathrm{CHs}$ to trigger attacks. Therefore, during both the set-up phase and the steady-state phase, the proposed secure routing is resilient and robust to sinkhole and selective forwarding attacks. Together with round rotating mechanism and digital signature scheme, the routing protocol is resilient to hello flood attacks to $\mathrm{CHs}$. Because the $\mathrm{CHs}$ being attacked are capable to ignore all the communication packets with bogus node ID or bogus digital signature.

\section{Protocol Evaluation}

In this section, we focus on the energy consumption spent on message propagation and computation. We use the network simulator OMNeT++ [19] to simulate the proposed protocol.

\section{A. Message Size}

We do the quantitative calculation in this subsection. In the proposed secure routing protocol, the total message packet size of during the transmissions which is added in formula (3) in section $\mathrm{V}$ equals to

$$
\left|I D_{j}\right|+\left|t_{i}\right|+|M|+\left|\sigma_{j}\right|+\left|h\left(M|| t_{i}|| \theta\right)\right| .
$$

Where, $\left|h\left(M \| t_{i}|| \theta\right)\right|$ is a hash value, which is 20 bytes when SHA-1 is used. $\left|I D_{j}\right|+\left|t_{i}\right|$ are very small like 2 bytes by each, and $|M|$ is assumed as 20 bytes. The total message size of a transmission packet is $44+\left|\sigma_{j}\right|$ bytes, whereas, $\left|\sigma_{j}\right|$ is variable. For example, when using the Tate pairing |14|, the elliptic curve $\mathbb{E}$ is defined over $\mathbb{F}_{p}$, the order $q$ of $\mathbb{G}_{1}$ and $\mathbb{G}_{2}$ is a 160 -bit prime. Moreover, $p$ could be a 512-bit prime to achieve higher level of security as 1024-bit RSA [5], and $\mathbb{G}$ is a $q$-order multiplicative subgroup of the finite field $\mathbb{F}_{p^{2}}^{*}$.

We compare the proposed secure routing protocol with two other existing secure routing protocols which are using symmetric key management, SecLEACH [12] and Multi-level $\mu$ Tesla based protocol [20]. Figure 2 shows the total message sizes in different protocols for data transmission, concerning the number of sensor nodes. We can see that the proposed ID-based secure routing protocol has smaller message size than multi-level $\mu$ Tesla based protocol. At the same time, it generates larger message size as compared to SecLEACH, however, the orphan node problem is fully solved in the proposed ID-based secure routing protocol. 


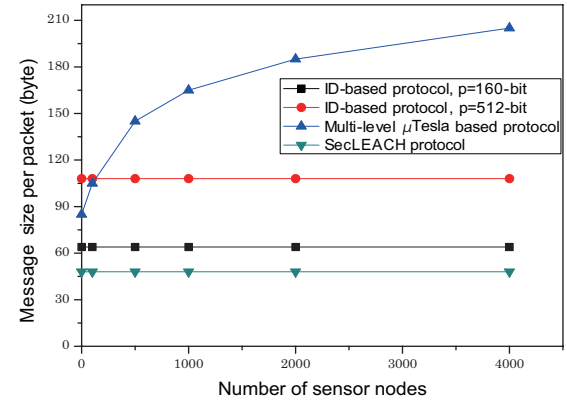

Fig. 2. Message packet size

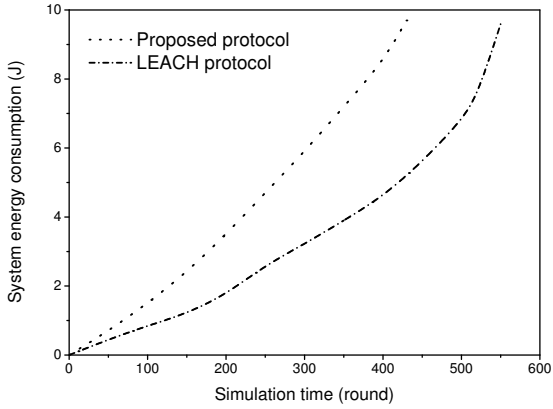

Fig. 3. Energy consumption in the system

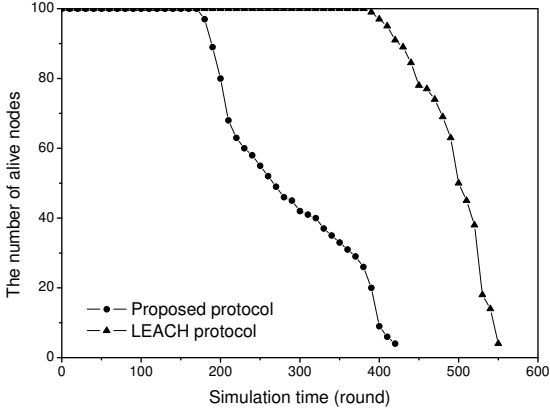

Fig. 4. The number of alive nodes

\section{B. Simulation Results}

Comprehending the extra energy consumption by the auxiliary security overhead and prolonging the network lifetime are essential in the proposed secure routing protocol. In order to evaluate the energy consumption of the security overhead in the proposed protocol, we consider two metrics for the performance evaluation: system energy consumption and the number of alive nodes. For the performance evaluation, we compare the proposed protocol with LEACH protocol [8].

In the simulation experiments, we use a network where 100 nodes were randomly distributed in a $100 \mathrm{~m} \times 100 \mathrm{~m}$ area, with a fixed BS located near the sensing field, and all sensor nodes periodically sense events and transmit the data packet to the BS. The initial energy of each sensor node is $0.5 \mathrm{~J}$, and the BS has unlimited energy. Meanwhile, we set the proper desired percentage of $\mathrm{CH}$ nodes $\rho=10 \%$ during one round. The communication energy parameter, electronics energy and computation cost are set as: $E_{\text {elec }}=50 \mathrm{~nJ} / \mathrm{bit}$, transmit amplifier is set as: $E_{a m p}=100 \mathrm{pJ} / \mathrm{bit} / \mathrm{m}^{2}$, and the energy for data aggregation is set as: $E_{\text {aggr }}=5 \mathrm{~nJ} / \mathrm{bit}$. Furthermore, for computation cost for digital signature, the energy consumption of bilinear transformation and pairing is $E_{\text {pair }}=25.5 \mathrm{~nJ} /$ pair, and the energy consumption on hash operations is $E_{\text {hash }}=7.6 \mathrm{~nJ} /$ function. Moreover, the hop-wise energy consumption on sending a message is $\mid$ byte $\times 59.2 \mu \mathrm{J} \mid$, and the energy consumption on receiving a message is byte $\times 28.6 \mu \mathrm{J} \mid[8\|5\| \mid 21]$.

Figure 3 presents how the energy disseminates in the network system and is consumed by sensor nodes. Figure 4 shows the comparison of system lifetime using the proposed secure routing protocol versus the LEACH protocol [8]. The results demonstrate that the proposed protocol consumes energy faster than LEACH protocol, which indicates that, the sensor nodes in the proposed secure routing protocol exhaust faster, because of the communication overhead and the pairing computation cost for ID-based digital signature.

\section{CONCLUSion AND Future Work}

In this paper, we have introduced a novel secure routing protocol for cluster-based WSNs using ID-based digital signature. The proposed protocol is efficient in communication, and it achieves the security requirements in routing for cluster-based WSNs. However, the simulation results point out the issues in the proposed protocol that, the extra energy consumption by computation of the auxiliary security overhead is still large.

The future work is to improve the simulation experiments with other secure routing protocols for better results, and improve the protocol in energy efficiency with pairing.

\section{REFERENCES}

[1] K. Römer and F. Mattern, "The Design Space of Wireless Sensor Networks," IEEE Wireless Communications, vol. 11, no. 6, 2004.

[2] A. A. Abbasi and M. Younis, "A survey on clustering algorithms for wireless sensor networks," Computer Communications, vol. 30, no. 14$15,2007$.

[3] I. F. Akyildiz, W. Su et al., "Wireless sensor networks: a survey," Computer Networks, vol. 38, no. 4, 2002.

[4] J. Ibriq and I. Mahgoub, "Cluster-Based Routing in Wireless Sensor Networks: Issues and Challenges," in Proc. of SPECTS'04, 2004.

[5] D. Boneh and M. Franklin, "Identity-Based Encryption from the Weil Pairing," in Adv. in Cryptology - CRYPTO 2001, LNCS, 2001, vol. 2139.

[6] A. Shamir, "Identity-Based Cryptosystems and Signature Schemes," in Adv. in Cryptology - CRYPTO'84, LNCS, 1985, vol. 196.

[7] F. Hess, "Efficient Identity Based Signature Schemes Based on Pairings," in Selected Areas in Cryptography - SAC 2002, LNCS, 2003, vol. 2595.

[8] W. R. Heinzelman, A. Chandrakasan, and H. Balakrishnan, "EnergyEfficient Communication Protocol for Wireless Microsensor Networks," in Proc. of HICSS'O0, 2000.

[9] S. Lindsey and C. Raghavendra, "PEGASIS: Power-efficient gathering in sensor information systems," in 2002 IEEE Aerospace Conf. Proc., vol. 3, 2002.

[10] S. Yi, J. Heo et al., "PEACH: Power-efficient and adaptive clustering hierarchy protocol for wireless sensor networks," Computer Communications, vol. 30, no. 14-15, 2007

[11] A. Perrig, R. Szewczyk et al., "SPINS: security protocols for sensor networks," Wireless Networks, vol. 8, 2002.

[12] L. B. Oliveira, A. Ferreira et al., "SecLEACH-On the security of clustered sensor networks," Signal Processing, vol. 87, 2007.

[13] P. Banerjee, D. Jacobson, and S. Lahiri, "Security and performance analysis of a secure clustering protocol for sensor networks," in Proc. of IEEE NCA'07, 2007.

[14] P. Barreto, H. Kim et al., "Efficient Algorithms for Pairing-Based Cryptosystems," in Adv. in Cryptology - CRYPTO 2002, LNCS, 2002, vol. 2442.

[15] D. W. Carman, "New Directions in Sensor Network Key Management," Int'l Journal of Distributed Sensor Networks, vol. 1, 2005.

[16] K. S. McCurley, "The discrete Logarithm Problem," in Proc. of Symp. in Applied Mathematics, PCS, 1990, vol. 42.

[17] C. Karlof and D. Wagner, "Secure routing in wireless sensor networks: attacks and countermeasures," Ad Hoc Networks, vol. 1, no. 2-3, 2003.

[18] Secure Hash Standard, National Institute of Standards and Technology (NIST), Federal Info. Processing Standards Publication 180-1, 1995.

[19] "OMNeT++," OMNeT++ Community. url: http://www.omnetpp.org/

[20] D. Liu and P. Ning, "Multilevel $\mu$ TESLA: Broadcast authentication for distributed sensor networks," ACM Trans. on Embedded Computing Systems, vol. 3, 2004.

[21] K. Ren, W. Lou et al., "On Broadcast Authentication in Wireless Sensor Networks," IEEE Trans. on Wireless Communications, vol. 6, no. 11, 2007. 